\title{
Review Article \\ Systemic Oxidative Stress Biomarkers in Chronic Periodontitis: A Meta-Analysis
}

\author{
Zhiqiang Liu, ${ }^{1,2}$ Yan Liu, ${ }^{1,2}$ Yiqing Song, ${ }^{3}$ Xi Zhang, ${ }^{4}$ Songlin Wang, and Zuomin Wang ${ }^{1,2}$ \\ ${ }^{1}$ Department of Stomatology, Beijing ChaoYang Hospital Affiliated to Capital Medical University, Beijing 100020, China \\ ${ }^{2}$ Beijing Key Laboratory of Tooth Regeneration and Function Reconstruction, Capital Medical University School of Stomatology, \\ Beijing 100050, China \\ ${ }^{3}$ Department of Epidemiology, Indiana University Richard M. Fairbanks School of Public Health, Indianapolis, IN 46202, USA \\ ${ }^{4}$ Institute of Vascular Medicine, Peking University Third Hospital, Beijing 100191, China \\ ${ }^{5}$ Laboratory of Gene Therapy and Tooth Regeneration, Beijing Key Laboratory of Tooth Regeneration and Function Reconstruction, \\ Capital Medical University School of Stomatology, Beijing 100050, China
}

Correspondence should be addressed to Songlin Wang; slwang@ccmu.edu.cn and Zuomin Wang; wzuomin@gmail.com

Received 2 August 2014; Revised 10 October 2014; Accepted 13 October 2014; Published 16 November 2014

Academic Editor: Seul-Ki Jeong

Copyright (c) 2014 Zhiqiang Liu et al. This is an open access article distributed under the Creative Commons Attribution License, which permits unrestricted use, distribution, and reproduction in any medium, provided the original work is properly cited.

\begin{abstract}
Oxidative stress biomarkers have been observed in peripheral blood of chronic periodontitis patients; however, their associations with periodontitis were not consistent. This meta-analysis was performed to clarify the associations between chronic periodontitis and oxidative biomarkers in systemic circulation. Electronic searches of PubMed and Embase databases were performed until October 2014 and articles were selected to meet inclusion criteria. Data of oxidative biomarkers levels in peripheral blood of periodontitis patients and periodontal healthy controls were extracted to calculate standardized mean differences (SMDs) and 95\% confidence intervals (CIs) by using random-effects model. Of 31 eligible articles, 16 articles with available data were included in meta-analysis. Our results showed that periodontitis patients had significantly lower levels of total antioxidant capacity (SMD $=-2.02 ; 95 \% \mathrm{CI}:-3.08,-0.96 ; P=0.000)$ and higher levels of malondialdehyde (SMD $=0.99 ; 95 \% \mathrm{CI}: 0.12,1.86 ; P=0.026)$ and nitric oxide (SMD $=4.98 ; 95 \%$ CI: $2.33,7.63 ; P=0.000$ ) than periodontal healthy control. Superoxide dismutase levels between two groups were not significantly different $(\mathrm{SMD}=-1.72 ; 95 \% \mathrm{CI}:-3.50,0.07 ; P=0.059)$. In conclusion, our meta-analysis showed that chronic periodontitis is significantly associated with circulating levels of three oxidative stress biomarkers, indicating a role of chronic periodontitis in systemic diseases.
\end{abstract}

\section{Introduction}

Chronic periodontitis, characterized by inflammation and destruction of periodontal supporting tissues, is one of the most common oral diseases worldwide. Over $47 \%$ of American people had chronic periodontitis [1], and the prevalence is even higher in developing countries [2]. Chronic periodontitis is initially caused by various hyperresponsive and destructive products of immune response stimulated by microbial plaque around the gingival margin.

In the pathogenesis of periodontitis, polymorphonuclear leukocytes (PMN) act as the primary mediators of the host response against proliferating periodontal pathogenic microorganisms. Activated PMN produce a large amount of reactive oxygen species (ROS) and result in destruction of periodontal tissues $[3,4]$. There is some suggestive evidence that periodontal inflammation might be associated with systemic oxidative stress. Recently, abundant evidence has shown that periodontal diseases were highly associated with several inflammation-related systemic diseases, such as chronic respiratory diseases [5], cardiovascular disease [6], and diabetes mellitus [7]. Oxidative stress plays an important role in the pathogenesis of these diseases [8-11]. It has been hypothesized that oxidative stress arising from periodontal lesions may be an important cause of systemic inflammation. Some but not all epidemiological studies have shown that biomarkers levels of oxidative stress in the peripheral blood of periodontitis patients were different from periodontal 
healthy subjects [12-14]. However, different levels of oxidative stress biomarkers had been detected in peripheral blood of chronic periodontitis patients in different studies, and also their findings were not consistent.

To test the hypothesis that chronic periodontitis is associated with systemic oxidative stress, we therefore carried out a meta-analysis of all published relevant cross-sectional, case control, and intervention studies to provide a comprehensive and quantitative synthesis of accumulative evidence.

\section{Methods}

2.1. Search Strategy. A literature search was conducted using the PubMed and Embase databases update to October 2014. The search strategy was as follows: (1) keywords "oxidat", "antioxidat"," "oxidant", "antioxidant"," "redox," "reactive oxygen species," "ROS," and common used oxidative stress biomarkers keywords "total antioxidant capacity," "TAOC," "total oxidant status," "TOS," "total antioxidant status," "TAS," "oxidative stress index," "OSI," "nitric oxide," "malondialdehyde," "MDA," "superoxide dismutase," "SOD," "reactive oxygen metabolites," "ROM," "glutathione," "GSH," "glutathione peroxidase," "GPx," "8-hydroxy-deoxyguanosine," " $8-\mathrm{OHdG}$ ", and "catalase" were connected by Boolean operator "OR"; (2) keywords "serum," "plasma," and "blood" were connected by Boolean operator "OR"; (3) the above search results and the keyword "periodont" were connected by Boolean operator "AND”. All keywords were restricted in title or abstract without the language limitation.

2.2. Study Selection. Two reviewers scanned the titles and abstracts of all above searched articles independently to look for the relevant studies reporting the biomarkers levels of oxidative stress in periodontitis patients and healthy controls. The inclusion criteria were as follows: (1) study subjects were human adults; (2) 7.both periodontitis patients group and periodontal healthy control group were included in the study; (3) at least one oxidative stress biomarker was measured in peripheral blood samples; (4) language was English. If any of above criteria could not be identified only through title and abstract, full text of the article was reviewed.

Full texts of all above included articles were then reviewed for a further selection. The exclusion criteria were as follows: (1) smokers and/or subjects with systemic diseases were not excluded from periodontitis group and/or periodontal healthy control group; (2) biomarkers levels were only shown by histogram with no detailed data; (3) if two articles have the same study population, only the latest published article was selected; (4) oxidative biomarkers were only measured in red blood cells or lymphocytes. Any disagreements were discussed for a consensus.

2.3. Data Extraction. Data were extracted by two reviewers independently. Mean $\pm \mathrm{SD}$, median (min-max), or median $(25 \%-75 \%)$ of peripheral blood oxidative stress biomarkers in periodontitis patients and periodontal healthy control groups was extracted. If it was a clinical intervention study, we only extracted the baseline data before periodontal treatment.
When there were more than two groups in one study, we only focused on the data of systemic healthy periodontitis patients and healthy control.

2.4. Quality Assessment. Newcastle-Ottawa Quality Assessment Scale (NOS) (http://www.ohri.ca/programs/clinical_ epidemiology/oxford.asp) was used to assess the methodological quality of all the included nonrandomized studies. The NOS for case control studies includes three domains (selection, comparability, and outcomes) and eight items. A study can be awarded a maximum of one star for each numbered item within the selection and exposure categories. A maximum of two stars can be given for comparability. Clinical intervention studies included in this study were assessed with the same scale as case control studies, because only baseline data were focused on and extracted in our analysis. Two reviewers worked independently, and discrepancies were resolved by discussion.

2.5. Statistical Analyses. Meta-analyses were conducted to summarize the differences levels of oxidative stress biomarkers between periodontitis patients and healthy controls, if there were 3 more studies reporting the same biomarker measurement and the biomarker levels were expressed by mean $\pm \mathrm{SD}$.

Since the included studies used different assay methods and units for the same biomarker, we calculated the standardized mean difference (SMD) and 95\% confidence interval (CI) as a summary statistic in meta-analysis for the difference of the levels of each biomarker between periodontitis patients and periodontal healthy controls. SMD is the mean divided by the standard deviation of a difference in each biomarker between patients and controls and can be seen as the mean difference that would have been obtained if all data were transformed to a scale where the standard deviation within groups was equal to 1.0 .

Heterogeneity among studies was assessed by $Q$ test, which indicated a significant heterogeneity if $P$ value $<0.05$. We also quantified the extent of heterogeneity with the $I^{2}$ value, where the percentages of $I^{2} 25-50 \%, 50-75 \%$, and $>75 \%$ indicate low, medium, and high heterogeneity, respectively. A random-effects model was used to calculate SMD when there was a significant heterogeneity; otherwise a fixed-effects model was used. Statistical analyses were carried out using the Stata statistical software version 12.0 (Stata, College Station, TX, USA).

\section{Results}

3.1. Study Selection and Characteristics. A total of 297 and 294 articles were separately screened based on electronic search from PubMed and Embase database. 329 articles were left after duplicates were removed. Only 31 relevant articles published from December 2002 to September 2014, including 21 cross-sectional or case-control design studies and 10 clinical intervention studies, were finally selected after a title or abstract review and a full text review according to the exclusion and inclusion criteria. At last, 16 articles were 


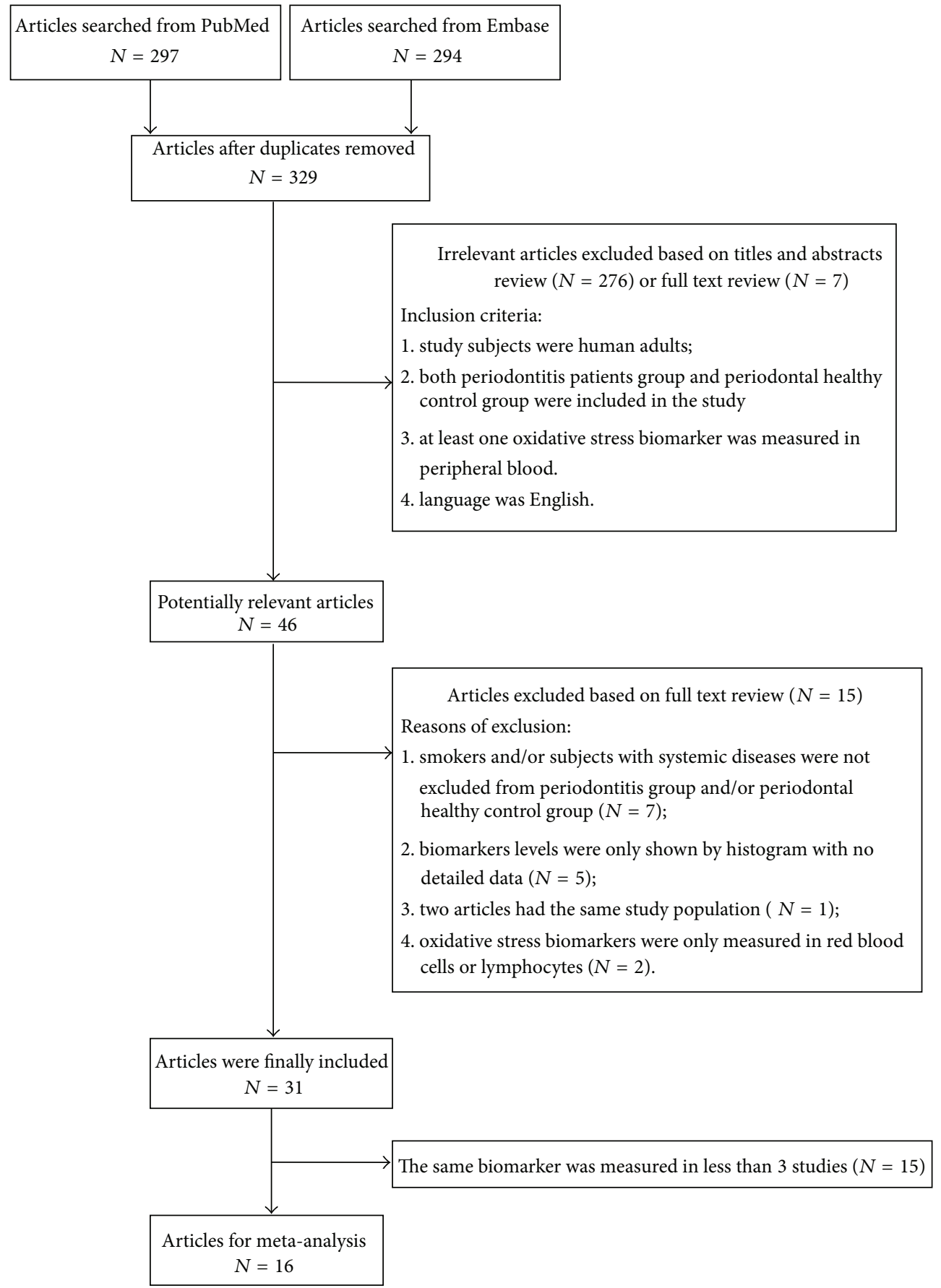

FIGURE 1: Flow chart of the process of study selection.

included in meta-analysis. The process of study selection and the specific information of these studies were depicted in Figure 1 and basic characteristics of all included studies were summarized in Table 1.

3.2. Quality Assessment. The results of quality assessment with NOS were shown in Supplemental Table 1 available online at http://dx.doi.org/10.1155/2014/931083. All included studies had middle or high quality. Seven studies were given
6 stars, 20 studies were given 7 stars, and 4 studies were given 8 stars. Almost all studies met the NOS criteria for case definition and had good representativeness of cases. However, for selection of controls, almost all studies derived from the same population as the cases, but hospital controls were used. In all studies, controls had good periodontal status when they were included in the studies, but these studies did not explicitly state that controls had no history of periodontitis except 4 studies. 


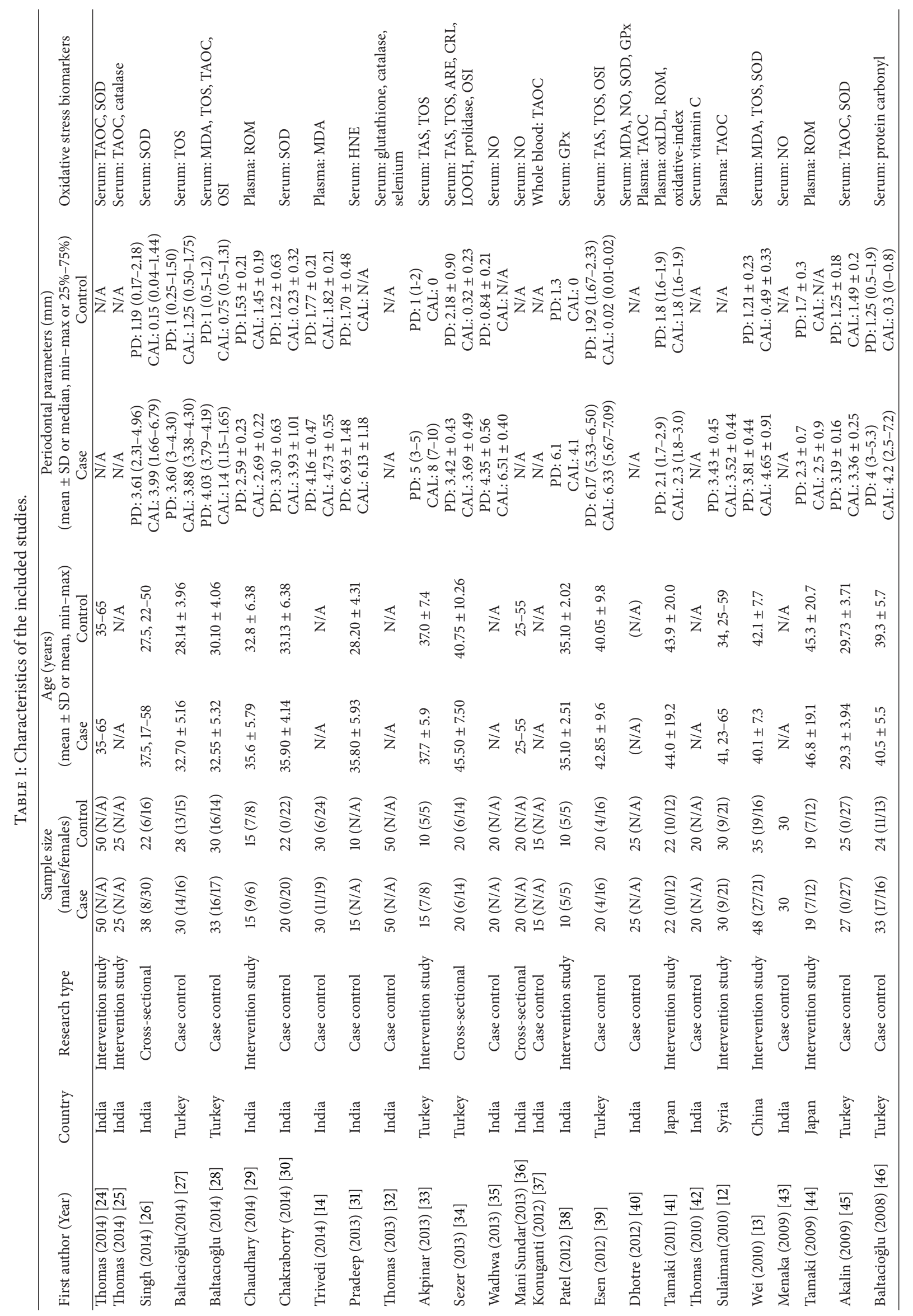




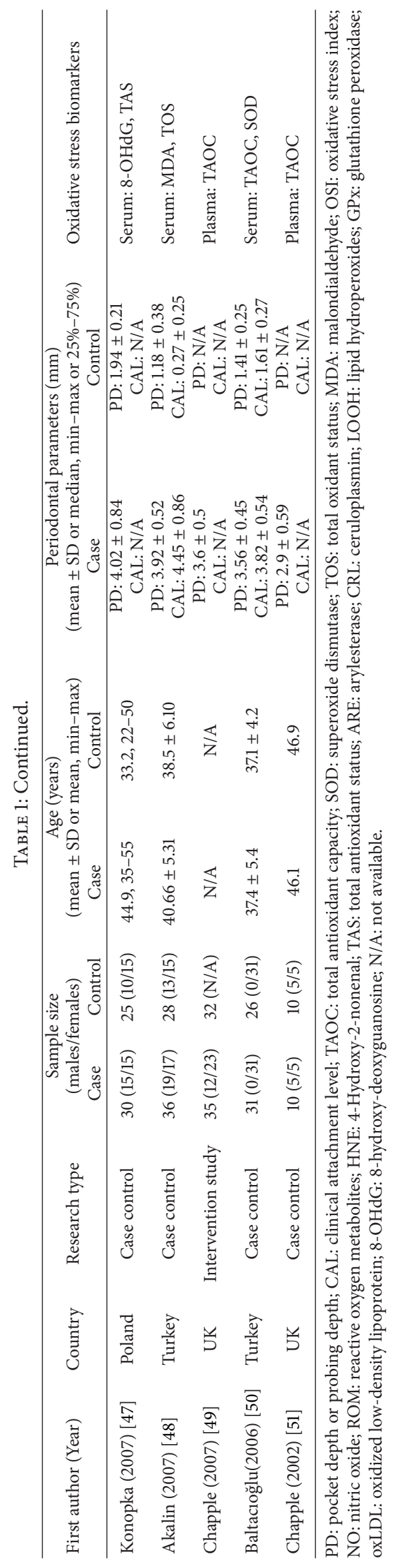


Smoking and systemic diseases are the main confounders to induce systemic oxidative stress. Because studies with smokers and subjects that had systemic diseases in both groups were excluded during study selection procedure, all included studies had good comparability of cases and controls and were given two stars.

For exposure, oxidative biomarkers levels in case and control groups were all determined in laboratory and had secure record. Response rate was the same for both groups. So all included studies were given 3 stars.

3.3. Oxidative Biomarkers in the Meta-Analysis. Meta-analyses of four oxidative biomarkers including total antioxidant capacity (TAOC), malondialdehyde (MDA), superoxide dismutase (SOD), and nitric oxide (NO) were performed with random-effects model. Significant heterogeneity existed among studies (all $P<0.001$ and $I^{2}>90 \%$ ). The results were presented in Figure 2.

In total, TAOC was determined in 9 studies. 7 studies showed lower TAOC levels in periodontitis group than in periodontal health group, and the other two showed a nonsignificant difference between two groups. Meta-analysis results show that periodontitis patients had lower TAOC levels than periodontal healthy controls $(\mathrm{SMD}=-2.02 ; 95 \%$ CI: $-3.08,-0.96 ; P=0.000)$.

MDA was determined in 5 studies; 2 studies showed higher levels in periodontitis group than in periodontal health group, and the other three showed a nonsignificant difference between two groups. Meta-analysis results show that periodontitis patients had higher MDA levels than periodontal healthy controls (SMD $=0.99$; 95\% CI: 0.12, 1.86; $P=0.026)$.

Of 6 articles that reported SOD, 5 showed lower SOD levels in periodontitis group than in periodontal health group, and the other one showed an opposite significant difference. The final meta-analysis results show nonsignificant difference between two groups (SMD $=-1.72 ; 95 \% \mathrm{CI}:-3.50,0.07 ; P=$ 0.059).

All the 4 studies that determined NO showed higher NO levels in periodontitis group than in periodontal health group, and meta-analysis shows the same results $(\mathrm{SMD}=$ 4.98; 95\% CI: 2.33, 7.63; $P=0.000)$.

\section{Discussion}

This meta-analysis systematically summarized the results of 16 independent studies from different countries and suggested that oxidative stress biomarkers TAOC levels in peripheral blood were lower and MDA and NO levels in peripheral blood were higher in periodontitis patients than healthy subjects, which indicated an elevation in systemic oxidative stress status in periodontitis patients. However, SOD levels in peripheral blood were not significantly different between periodontitis patients and healthy subjects. Taken together, these results clearly show that chronic periodontitis is significantly related to some but not all markers of oxidative stress status of systemic circulation.

There were no well-validated biomarkers of oxidative stress and various biomarkers were used in different studies.
Antioxidants, enzymes, and the oxidation products of protein, lipids, and DNA were widely used to indicate the oxidative status and also acted as oxidative stress biomarkers. Most of biomarkers were only measured in one or two studies of the 31 included relevant articles. Our meta-analysis focused on biomarkers that were measured and reported at least in three studies, which ensure enough statistical power of meta-analysis. Systemic diseases were associated with ROS $[9,10,15,16]$, and cigarette can produce thousands of kinds of ROS molecules [17], so studies with patients of systemic disease or smokers were excluded.

Our results showed that TAOC levels decreased and MDA and NO levels increased in the peripheral blood of chronic periodontitis patients. MDA is the principal product of polyunsaturated fatty acid peroxidation that can indicate the increase of oxidative stress [18]. NO is a short-lived reactive free radical and is synthesized by the oxidative process of the guanidine of the amino acid L-arginine [19]. These suggested that periodontal inflammation might trigger systemic oxidative stress. PMN activation in peripheral blood could result in the increment of ROS in circulation. Matthews et al. demonstrated that peripheral neutrophils (a subgroup of PMN) from chronic periodontitis patients showed an increase of extracellular ROS release in vitro without exogenous stimulation [20]. Dias et al. found that proinflammatory cytokines such as IL-8, GM-CSF, and IFN$\alpha$ increased in the plasma of periodontitis patients. These cytokines in periodontitis patients were more effective in stimulating neutrophil superoxide production than those in healthy controls [21]. Periodontitis may cause oxidative damage to multiple distant organs. An animal research showed that excessive production of lipid peroxide following periodontal inflammation was involved in oxidative DNA damage of the brain, heart, liver, and kidney in rats [22].

$\mathrm{SOD}$ is an enzyme that catalyses the dismutation of $\mathrm{O}_{2}{ }^{--}$ (a ROS molecular) to $\mathrm{H}_{2} \mathrm{O}_{2}$ and $\mathrm{O}_{2}$ [23]. Although five studies showed lower SOD levels in periodontitis group than in periodontal health group and the other one showed an opposite significant difference, all these studies suggested the same conclusion that periodontitis could alter systemic SOD levels. When SOD levels were found lower in periodontitis patients, it can be explained as more superoxides were induced as periodontal inflammation. More SOD would be consumed and cause SOD level decrease. On the other hand, when SOD levels were found higher in periodontitis patients, it can be explained as that more SOD were produced to afford biological protection against increased superoxide generation during periodontal inflammation. However, our meta-analysis result of six studies showed that SOD levels had no significant difference between periodontitis group and healthy subjects.

This meta-analysis provides overall evidence on the relation between available biomarkers of oxidative stress and chronic periodontitis from human peripheral blood samples. However, there are some limitations caused by study heterogeneity, such as designs, sample sizes, and methods of biomarker measurement. There was a remarkable variability among selected studies regarding the types of oxidative stress biomarkers. In total, more than a dozen different biomarkers 


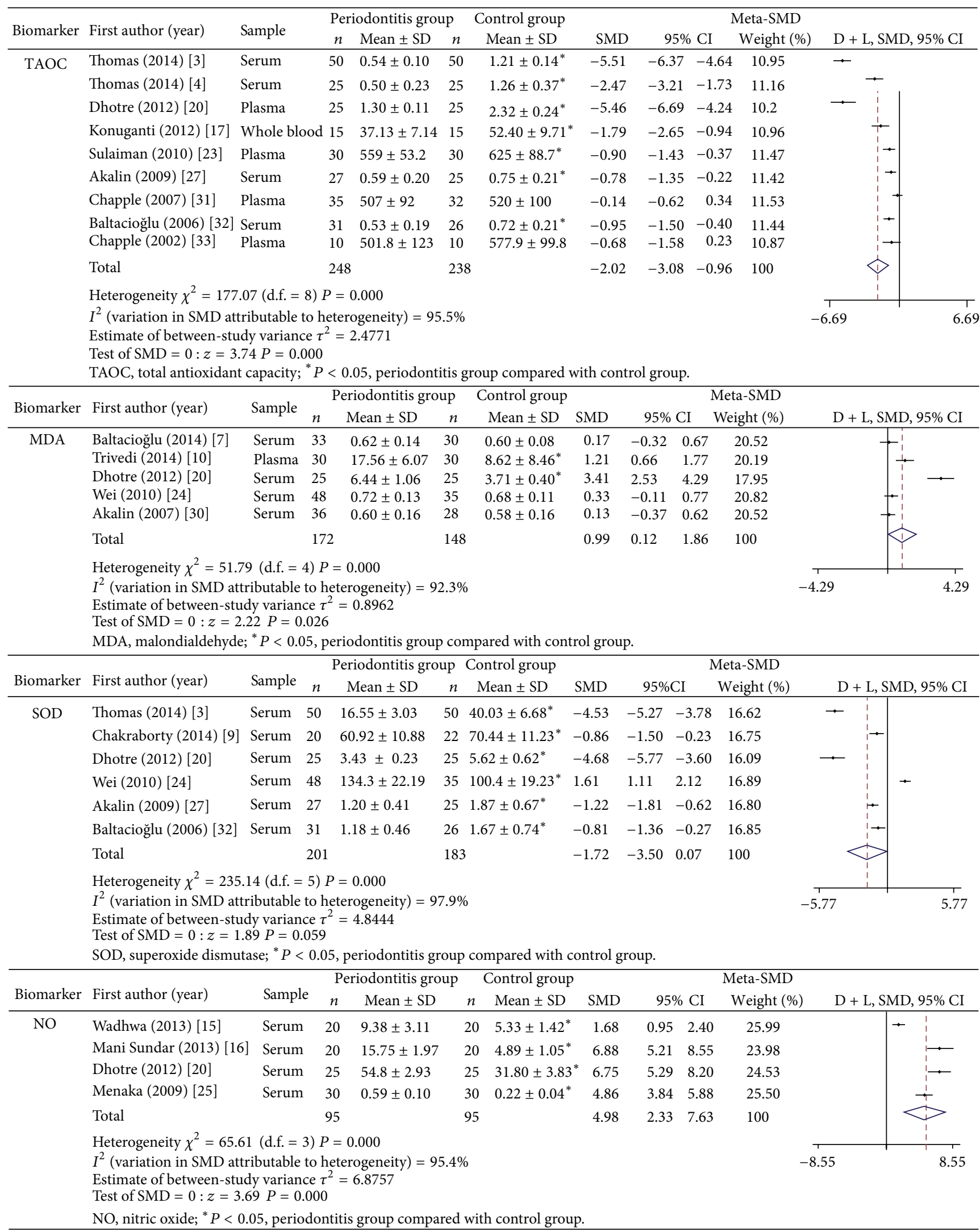

FIGURE 2: Meta-analysis of oxidative stress biomarkers in peripheral blood of periodontitis patients and controls. 
were detected in all these selected studies. All biomarkers conducted in meta-analysis were only detected in four to nine studies. Less studies numbers and small sample size may reduce the statistical power of analysis. On the another hand, different measurement methods were used in different studies even for the same oxidative stress biomarker, so that the biomarker levels were in different orders of magnitude or have different units in different studies. Effect scale SMD was used in this meta-analysis as its advantages could reduce this discrepancy. Random-effects model was applied for SMD estimation of biomarkers with high heterogeneity (all $I^{2}>$ $90 \%$ ) among studies. The heterogeneity may also be induced by different definition of chronic periodontitis, different study populations (sex and age), and different biological specimens (serum, plasma, or whole blood) among different studies. Different study designs (cross-sectional, case control, or interventional study) may also produce the heterogeneity. In addition, inherent limitations of the cross-sectional and case control study designs do not allow inferences about causality concerning the association.

\section{Conclusion}

Our meta-analysis results suggested that oxidative biomarkers TAOC, MDA, and NO levels from peripheral blood samples were significantly different between periodontitis patients and healthy subjects. This evidence suggested that chronic periodontitis was associated with systemic oxidative stress in human bodies. Our findings further indicated that clinical intervention of periodontitis may be beneficial for periodontitis patients' systemic oxidative stress control and reduce its potential effect to systemic diseases. Prospective studies and randomized trials are needed to verify this hypothesis in the future.

\section{Conflict of Interests}

The authors declare that there is no conflict of interests regarding the publication of this paper.

\section{Authors' Contribution}

Yan Liu co-first author contributed equally to this paper.

\section{Acknowledgments}

This meta-analysis was supported by the National Natural Science Foundation of China (81271158) and Beijing Science and Technology Program Fund (Z131100006813027). The funding organizations played no role in the collection, analysis, and interpretation of the data or in the preparation, review, or approval of the paper.

\section{References}

[1] P. I. Eke, B. A. Dye, L. Wei, G. O. Thornton-Evans, and R. J. Genco, "Prevalence of periodontitis in adults in the united states: 2009 and 2010," Journal of Dental Research, vol. 91, no. 10, pp. 914-920, 2012.
[2] E. F. Corbet, "Periodontal diseases in Asians," Journal of the International Academy of Periodontology, vol. 8, no. 4, pp. 136$144,2006$.

[3] C. F. Çanakçi, Y. Çiçek, and V. Çanakçi, "Reactive oxygen species and human inflammatory periodontal diseases," Biochemistry, vol. 70, no. 6, pp. 619-628, 2005.

[4] I. L. C. Chapple and J. B. Matthews, "The role of reactive oxygen and antioxidant species in periodontal tissue destruction," Periodontology 2000, vol. 43, no. 1, pp. 160-232, 2007.

[5] N. Sharma and H. Shamsuddin, "Association between respiratory disease in hospitalized patients and periodontal disease: a cross-sectional study," Journal of Periodontology, vol. 82, no. 8, pp. 1155-1160, 2011.

[6] X. Y. Ouyang, W. M. Xiao, Y. Chu, and S. Y. Zhou, "Influence of periodontal intervention therapy on risk of cardiovascular disease," Periodontology 2000, vol. 56, no. 1, pp. 227-257, 2011.

[7] T. B. Taiyeb-Ali, R. P. C. Raman, and R. D. Vaithilingam, "Relationship between periodontal disease and diabetes mellitus: an Asian perspective," Periodontology 2000, vol. 56, no. 1, pp. 258268, 2011.

[8] S. Loukides, P. Bakakos, and K. Kostikas, "Oxidative stress in patients with COPD," Current Drug Targets, vol. 12, no. 4, pp. 469-477, 2011.

[9] K. Stadler, "Oxidative stress in diabetes," Advances in Experimental Medicine and Biology, vol. 771, pp. 272-287, 2012.

[10] R. Lee, M. Margaritis, K. M. Channon, and C. Antoniades, "Evaluating oxidative stress in human cardiovascular disease: methodological aspects and considerations," Current Medicinal Chemistry, vol. 19, no. 16, pp. 2504-2520, 2012.

[11] R. M. Tuder and I. Petrache, "Pathogenesis of chronic obstructive pulmonary disease," Journal of Clinical Investigation, vol. 122, no. 8, pp. 2749-2755, 2012.

[12] A. E. A. Sulaiman and R. M. H. Shehadeh, "Assessment of total antioxidant capacity and the use of vitamin $\mathrm{C}$ in the treatment of non-smokers with chronic periodontitis," Journal of Periodontology, vol. 81, no. 11, pp. 1547-1554, 2010.

[13] D. Wei, X.-L. Zhang, Y.-Z. Wang, C.-X. Yang, and G. Chen, "Lipid peroxidation levels, total oxidant status and superoxide dismutase in serum, saliva and gingival crevicular fluid in chronic periodontitis patients before and after periodontal therapy," Australian Dental Journal, vol. 55, no. 1, pp. 70-78, 2010.

[14] S. Trivedi, N. Lal, A. A. Mahdi, M. Mittal, B. Singh, and S. Pandey, "Evaluation of antioxidant enzymes activity and malondialdehyde levels in patients with chronic periodontitis and diabetes mellitus," Journal of Periodontology, vol. 85, no. 5, pp. 713-720, 2014.

[15] M. Majzunova, I. Dovinova, M. Barancik, and J. Y. H. Chan, "Redox signaling in pathophysiology of hypertension," Journal of Biomedical Science, vol. 20, no. 1, article 69, 2013.

[16] T. Costacou, R. W. Evans, G. L. Schafer, and T. J. Orchard, "Oxidative stress and response in relation to coronary artery disease in type 1 diabetes," Diabetes Care, vol. 36, no. 11, pp. 3503-3509, 2013.

[17] D. F. Church and W. A. Pryor, "Free-radical chemistry of cigarette smoke and its toxicological implications," Environmental Health Perspectives, vol. 64, pp. 111-126, 1985.

[18] D. Del Rio, A. J. Stewart, and N. Pellegrini, "A review of recent studies on malondialdehyde as toxic molecule and biological marker of oxidative stress," Nutrition, Metabolism and Cardiovascular Diseases, vol. 15, no. 4, pp. 316-328, 2005. 
[19] S. Moncada and A. Higgs, "The L-arginine-nitric oxide pathway," The New England Journal of Medicine, vol. 329, no. 27, pp. 2002-2012, 1993.

[20] J. B. Matthews, H. J. Wright, A. Roberts, P. R. Cooper, and I. L. C. Chapple, "Hyperactivity and reactivity of peripheral blood neutrophils in chronic periodontitis," Clinical and Experimental Immunology, vol. 147, no. 2, pp. 255-264, 2007.

[21] I. H. K. Dias, J. B. Matthews, I. L. C. Chapple, H. J. Wright, C. R. Dunston, and H. R. Griffiths, "Activation of the neutrophil respiratory burst by plasma from periodontitis patients is mediated by pro-inflammatory cytokines," Journal of Clinical Periodontology, vol. 38, no. 1, pp. 1-7, 2011.

[22] T. Tomofuji, D. Ekuni, K. Irie et al., "Relationships between periodontal inflammation, lipid peroxide and oxidative damage of multiple organs in rats," Biomedical Research, vol. 32, no. 5, pp. 343-349, 2011.

[23] J. M. McCord and M. A. Edeas, "SOD, oxidative stress and human pathologies: a brief history and a future vision," Biomedicine and Pharmacotherapy, vol. 59, no. 4, pp. 139-142, 2005.

[24] B. Thomas, A. Rao, B. R. Prasad, and S. Kumari, "Serum levels of antioxidants and superoxide dismutase in periodontitis patients with diabetes type 2," Journal of Indian Society of Periodontology, vol. 18, no. 4, pp. 451-455, 2014.

[25] B. Thomas, S. M. Madani, B. R. Prasad, and S. Kumari, "Comparative evaluation of serum antioxidant levels in periodontally diseased patients: an interventional study," Contemporary Clinical Dentistry, vol. 5, no. 3, pp. 340-344, 2014.

[26] N. Singh, S. C. Narula, R. K. Sharma, S. Tewari, and P. K. Sehgal, "Vitamin e supplementation, superoxide dismutase status, and outcome of scaling and root planing in patients with chronic periodontitis: a randomized clinical trial," Journal of Periodontology, vol. 85, no. 2, pp. 242-249, 2014.

[27] E. Baltacioğlu, M. A. Kehribar, P. Yuva et al., "Total oxidant status and bone resorption biomarkers in serum and gingival crevicular fluid of patients with periodontitis," Journal of Periodontology, vol. 85, no. 2, pp. 317-326, 2014.

[28] E. Baltacıoğlu, P. Yuva, G. Aydın et al., "Lipid peroxidation levels and total oxidant/antioxidant status in serum and saliva from patients with chronic and aggressive periodontitis. Oxidative stress index: a new biomarker for periodontal disease?" Journal of Periodontology, vol. 85, no. 10, pp. 1432-1441, 2014.

[29] S. Chaudhary, T. M. Gowda, D. S. Mehta, and T. A. B. Kumar, "Comparative evaluation of plasma ROM levels in chronic periodontitis patients before and after non-surgical and surgical periodontal therapy: a clinical trial," Journal of Indian Society of Periodontology, vol. 18, no. 2, pp. 140-144, 2014.

[30] S. Chakraborty, S. Tewari, R. K. Sharma, S. C. Narula, P. S. Ghalaut, and V. Ghalaut, "Impact of iron deficiency anemia on chronic periodontitis and superoxide dismutase activity: a cross-sectional study," Journal of Periodontal \& Implant Science, vol. 44, no. 2, pp. 57-64, 2014.

[31] A. R. Pradeep, E. Agarwal, P. Bajaj, and N. S. Rao, "4Hydroxy-2-nonenal, an oxidative stress marker in crevicular fluid and serum in type 2 diabetes with chronic periodontitis," Contemporary Clinical Dentistry, vol. 4, no. 3, pp. 281-285, 2013.

[32] B. Thomas, A. Ramesh, S. Suresh, and B. R. Prasad, "A comparative evaluation of antioxidant enzymes and selenium in the serum of periodontitis patients with diabetes mellitus type 2," Contemporary Clinical Dentistry, vol. 4, no. 2, pp. 176-180, 2013.

[33] A. Akpinar, H. Toker, H. Ozdemir, V. Bostanci, and H. Aydin, "The effects of non-surgical periodontal therapy on oxidant and anti-oxidant status in smokers with chronic periodontitis," Archives of Oral Biology, vol. 58, no. 6, pp. 717-723, 2013.

[34] U. Sezer, K. Erciyas, K. Üstün et al., "Effect of chronic periodontitis on oxidative status in patients with rheumatoid arthritis," Journal of Periodontology, vol. 84, no. 6, pp. 785-792, 2013.

[35] D. Wadhwa, A. Bey, M. Hasija et al., "Determination of levels of nitric oxide in smoker and nonsmoker patients with chronic periodontitis," Journal of Periodontal \& Implant Science, vol. 43, no. 5, pp. 215-220, 2013.

[36] N. Mani Sundar, V. Krishnan, S. Krishnaraj, V. T. Hemalatha, and M. N. Alam, "Comparison of the salivary and the serum nitric oxide levels in chronic and aggressive periodontitis: a biochemical study," Journal of Clinical and Diagnostic Research, vol. 7, no. 6, pp. 1223-1227, 2013.

[37] K. Konuganti, H. Seshan, S. Zope, and W. D. Silvia, "A comparative evaluation of whole blood total antioxidant capacity using a novel nitroblue tetrazolium reduction test in patients with periodontitis and healthy subjects: a randomized, controlled trial," Journal of Indian Society of Periodontology, vol. 16, no. 4, pp. 620-622, 2012.

[38] S. P. Patel, N. S. Rao, and A. R. Pradeep, "Effect of nonsurgical periodontal therapy on crevicular fluid and serum glutathione peroxidase levels," Disease Markers, vol. 32, no. 1, pp. 1-7, 2012.

[39] C.. Esen, B. A. Alkan, M. Kirnap, Ö. Akgül, S. Işıkoğlu, and Ö. Erel, "The effects of chronic periodontitis and rheumatoid arthritis on serum and gingival crevicular fluid total antioxidant/oxidant status and oxidative stress index," Journal of Periodontology, vol. 83, no. 6, pp. 773-779, 2012.

[40] P. S. Dhotre, A. N. Suryakar, and R. B. Bhogade, "Oxidative stress in periodontitis," European Journal of General Medicine, vol. 9, no. 2, pp. 81-84, 2012.

[41] N. Tamaki, T. Tomofuji, D. Ekuni, R. Yamanaka, and M. Morita, "Periodontal treatment decreases plasma oxidized LDL level and oxidative stress," Clinical Oral Investigations, vol. 15, no. 6, pp. 953-958, 2011.

[42] B. Thomas, S. Kumari, K. Ramitha, and M. B. A. Kumari, "Comparative evaluation of micronutrient status in the serum of diabetes mellitus patients and healthy individuals with periodontitis," Journal of Indian Society of Periodontology, vol. 14, no. 1, pp. 46-49, 2010.

[43] K. B. Menaka, A. Ramesh, B. Thomas, and N. S. Kumari, "Estimation of nitric oxide as an inflammatory marker in periodontitis," Journal of Indian Society of Periodontology, vol. 13, no. 2, pp. 75-78, 2009.

[44] N. Tamaki, T. Tomofuji, D. Ekuni, R. Yamanaka, T. Yamamoto, and M. Morita, "Short-term effects of non-surgical periodontal treatment on plasma level of reactive oxygen metabolites in patients with chronic periodontitis," Journal of Periodontology, vol. 80, no. 6, pp. 901-906, 2009.

[45] F. A. Akalin, E. Baltacioǧlu, A. Alver, and E. Karabulut, “Total antioxidant capacity and superoxide dismutase activity levels in serum and gingival crevicular fluid in pregnant women with chronic periodontitis," Journal of Periodontology, vol. 80, no. 3, pp. 457-467, 2009.

[46] E. Baltacioğlu, F. A. Akalin, A. Alver, O. Deǧer, and E. Karabulut, "Protein carbonyl levels in serum and gingival crevicular fluid in patients with chronic periodontitis," Archives of Oral Biology, vol. 53, no. 8, pp. 716-722, 2008.

[47] T. Konopka, K. Król, W. Kopeć, and H. Gerber, "Total antioxidant status and 8-hydroxy-2' -deoxyguanosine levels in gingival and peripheral blood of periodontitis patients," Archivum 
Immunologiae et Therapiae Experimentalis, vol. 55, no. 6, pp. 417-422, 2007.

[48] F. A. Akalin, E. Baltacioğlu, A. Alver, and E. Karabulut, "Lipid peroxidation levels and total oxidant status in serum, saliva and gingival crevicular fluid in patients with chronic periodontitis," Journal of Clinical Periodontology, vol. 34, no. 7, pp. 558-565, 2007.

[49] I. L. C. Chapple, G. R. Brock, M. R. Milward, N. Ling, and J. B. Matthews, "Compromised GCF total antioxidant capacity in periodontitis: cause or effect?" Journal of Clinical Periodontology, vol. 34, no. 2, pp. 103-110, 2007.

[50] E. Baltacioğlu, F. A. Akalın, A. Alver, F. Balaban, M. Ünsal, and E. Karabulut, "Total antioxidant capacity and superoxide dismutase activity levels in serum and gingival crevicular fluid in post-menopausal women with chronic periodontitis," Journal of Clinical Periodontology, vol. 33, no. 6, pp. 385-392, 2006.

[51] I. L. C. Chapple, G. Brock, C. Eftimiadi, and J. B. Matthews, "Glutathione in gingival crevicular fluid and its relation to local antioxidant capacity in periodontal health and disease," Journal of Clinical Pathology: Molecular Pathology, vol. 55, no. 6, pp. 367-373, 2002. 


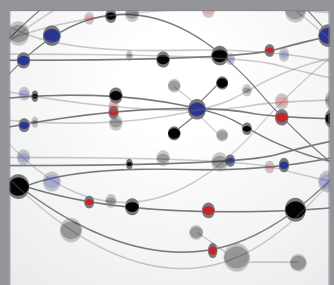

The Scientific World Journal
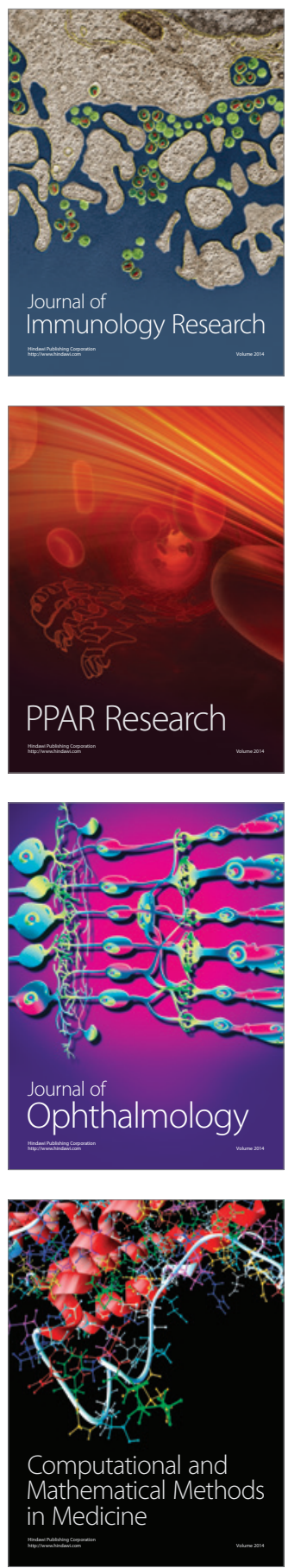

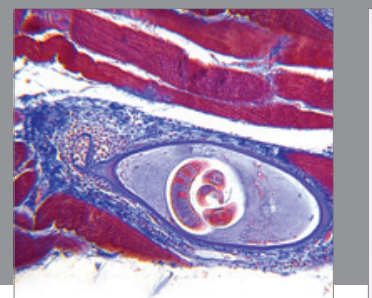

Gastroenterology

Research and Practice
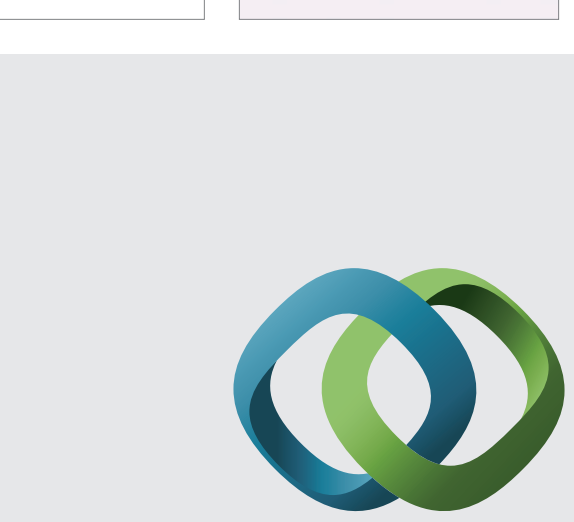

\section{Hindawi}

Submit your manuscripts at

http://www.hindawi.com
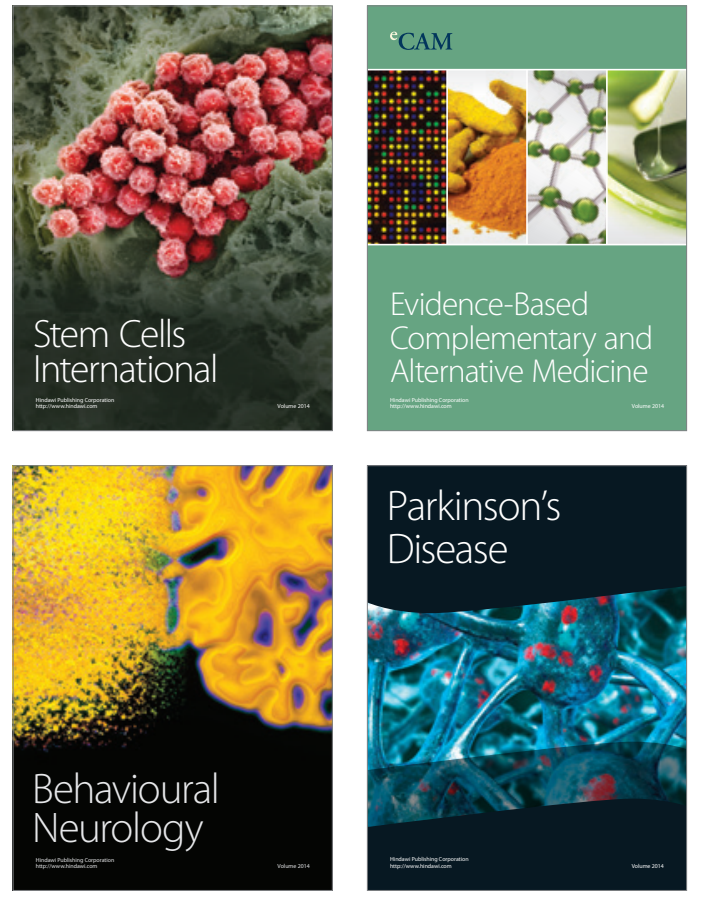
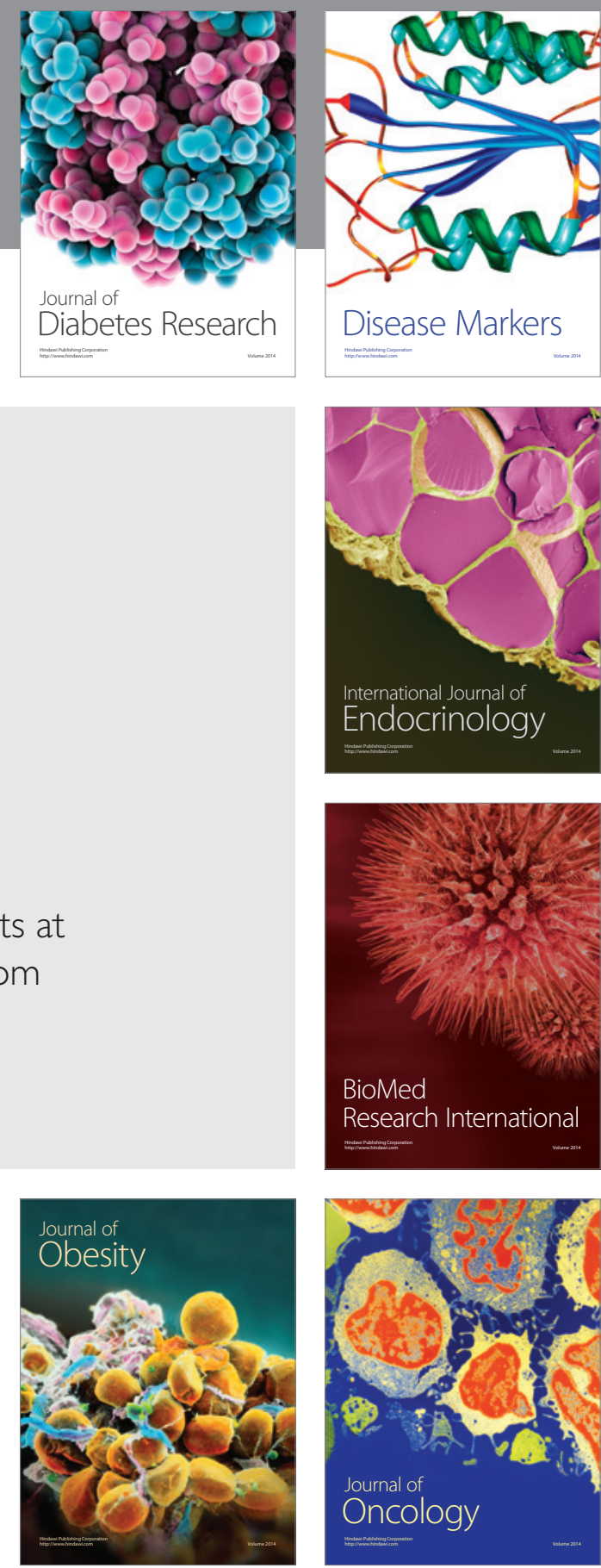

Disease Markers
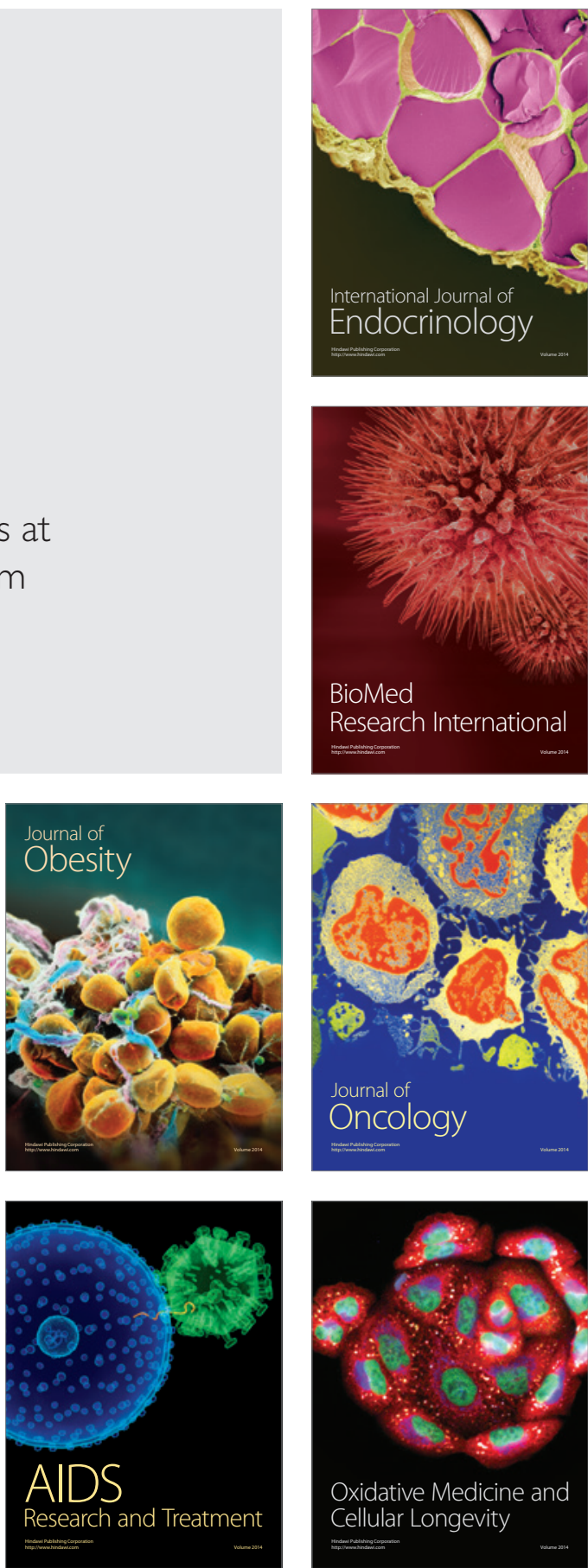